\title{
Investigating Premature Ignition of Thruster Pressure Cartridges by Mechanical Impact of Internal Components
}

\author{
Stephen S. Woods ${ }^{1}$ and Regor Saulsberry ${ }^{2}$ \\ NASA Johnson Space Center White Sands Test Facility, Las Cruces, NM, 88012
}

Pyrotechnic thruster pressure cartridges (TPCs) are used for aeroshell separation on a new NASA crew launch vehicle. The premature ignition concern was hypothesized based on the potential range of motion of the subassemblies, projected worst case accelerations, and the internal geometry that could subject propellant grains to mechanical impact sufficiently high for ignition. This possibility was investigated by fabricating a high-fidelity model of the suspected contact geometry, placing a representative amount of propellant in it, and impacting the propellant with a range of forces equivalent to and greater than the maximum possible during launch. Testing demonstrated that the likelihood of ignition is less than 1 in 1,000,000. The test apparatus, methodology, and results are described in this paper.

Nondestructive evaluation (NDE) during TPC acceptance testing indicated that internal assemblies moved during shock and vibration testing due to an internal bond anomaly. This caused concerns that the launch environment might produce the same movement and release propellant grains that might be prematurely ignited through impact or through electrostatic discharge (ESD) as grains vibrated against internal surfaces. Since a new lot could not be fabricated in time, a determination had to be made as to whether the lot was acceptable to fly. This paper discusses the analysis and impact testing used to address the potential impact issue and a separate paper addresses the ESD issue.

$\begin{array}{ll}\text { BOE } & =\text { Bureau of Explosives } \\ F t-l b f & =\text { foot pounds force } \\ g & =\text { acceleration due to gravity at the earth's surface } \\ H_{50} & =\text { Drop height at which } 50 \text { percent of events indicate a reaction } \\ J & =\text { Joules } \\ k g & =\text { kilogram } \\ m & =\text { mass } \\ m g & =\text { milligram } \\ N D E & =\text { Nondestructive Evaluation } \\ R M S & =\text { root mean square } \\ T P C & =\text { Thruster Pressure Cartridge } \\ v & =\text { velocity }\end{array}$

1 Senior Scientist, Laboratories Department/201 LD, NASA Johnson Space Center White Sands Test Facility, P.O. Box 20, Las Cruces, New Mexico 88004.

2 Project Manager, Laboratories Office/RF, P.O. Box 20, Las Cruces, NM 88004, Senior Member. 


\section{Introduction}

$\mathrm{T}$

HIS report documents the efforts, findings, and limitations of the investigation work for potential premature ignition of anomalous thruster pressure cartridges (TPC) by vibration-induced mechanical impact from loose internal components. TPCs are pyrotechnically powered devices that function as a powerful piston and are used for payload aeroshell separation. An anomalous state arises when component installation occurs before completion of qualification testing and nondestructive evaluation (NDE) of components from the same manufactured lot, and the subsequent results identify potential deficiencies.

Standard mechanical impact test techniques have been modified to duplicate component internal geometry to realistically simulate potential impact forces upon Hi-Temp ${ }^{\mathrm{TM}}{ }^{*}$ grains, an intermediate pyrotechnic in the TPC firing chain.

\section{Problem Description}

Pyrotechnic devices are subjected to rigorous testing and inspection to ensure operation under critical flight conditions. However, schedule demands may lead to TPC installation prior to the completion of NDE of samples. In one such instance, when the NDE results for TPCs from the same lot became available, there was evidence in several units that internal subassemblies and one of the pyrotechnic components, Hi-Temp, could be loose and free to move in volumes internal to the TPCs already installed on the vehicle.

\section{A. System Description}

From the outside, the TPCs resemble a steel cylinder $\sim 8$ in. long and a little more than an inch in diameter as shown in Fig. 1.
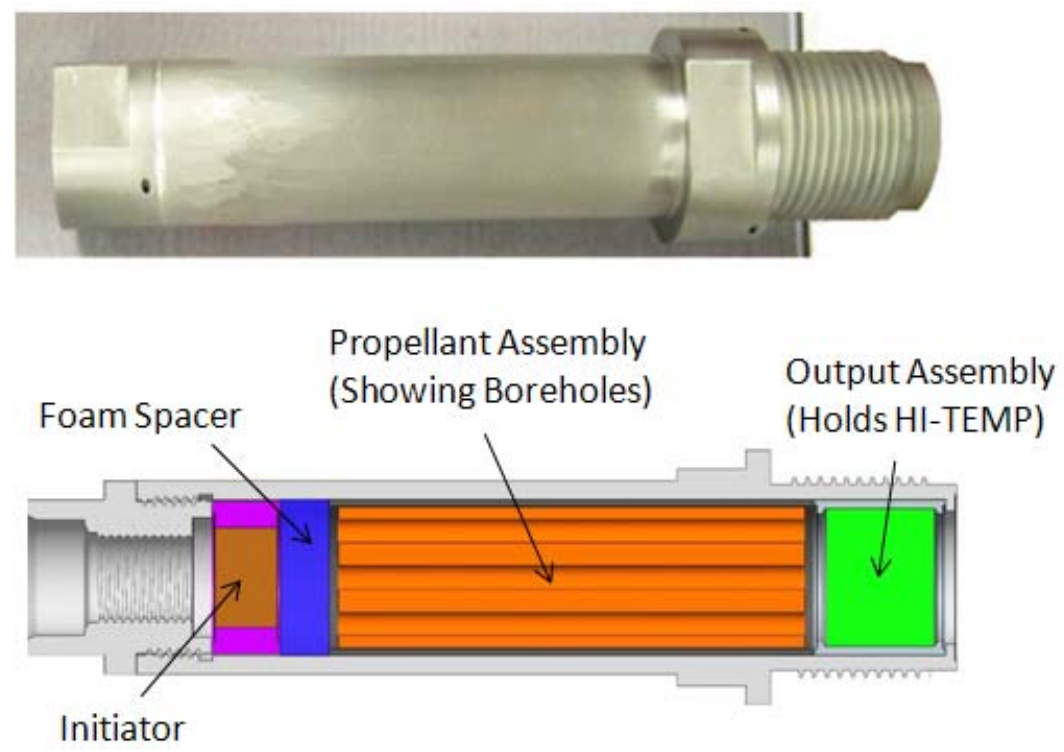

Figure 1. Thruster pressure cartridge.

Inside, and by position from left to right when viewed from the side, the TPC contains the initiator, an open cell polyurethane foam spacer, the propellant assembly, and the output assembly that holds the Hi-Temp (note that scale is only approximate). Both the propellant assembly and the output assembly are cylindrical and before gluing, readily slide within the case. The propellant assembly is affixed to the TPC shell by epoxy and the output assembly is corralled in place by the fixed position of the propellant assembly and an inner lip of the outer case. NDE evidence in some units examined after vibration environmental tests has indicated failure of the epoxy bonds between the propellant assembly and the case. This failure allows the propellant assembly to move because the foam spacer readily compresses to a relatively small volume with very little force. If the propellant assembly can move, then the output assembly can move. The same NDE provided views that further suggested Hi-Temp had been released from tears in the covers of the output assembly. If a unit was inadvertently installed on a launch vehicle in this state, and then subjected to launch, the TPC could be subjected to accelerations over a short period of time with

\footnotetext{
${ }^{*}$ Hi-Temp ${ }^{\mathrm{TM}}$ is a registered trademark of Hi-Temp Coatings Technology Company, Inc., Boxborough, Massachusetts.
} 
RMS values as high as 30 to $40 \mathrm{~g}$ depending on the launch vehicle. The high accelerations derived from the three sigma point in a distribution of vibrations that characteristic the launch environment. With the failure of the epoxy, the propellant assembly and output assembly could slide rapidly back and forth, and often slide together.

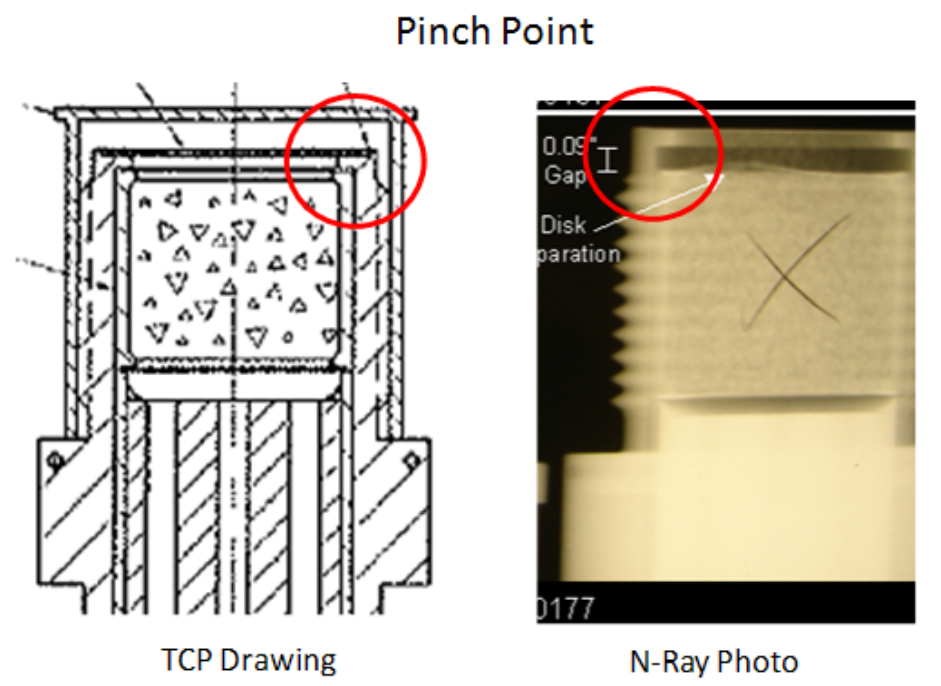

Figure 2. Schematic and N-Ray photograph showing view of pinch point within circle.

The output assembly possesses a steel case as does the retaining lip of the outer case. During vibration the case of the output assembly and the inner lip of the outer case can collide (Fig. 2), crushing any Hi-Temp grains caught in between.

\section{B. Impact Energies and Initiation}

Impact tests on Hi-Temp performed by UTEC ${ }^{1}$ using the BOE test method reported Bruceton ${ }^{2}$ test series results, $\mathrm{H}_{50}=5.08 \mathrm{in}$. for $10 \mathrm{mg}$ samples. The BOE Impact test applies a $3.63 \mathrm{~kg}$ weight dropped at heights varied in $1 \mathrm{in}$. increments. In this test, the previous drop height of 4 in. provides the no-reaction limit, which is determined (mass $\mathrm{x}$ maximum acceleration $x$ distance) to be an energy of 3.6 J. An analysis of the effects of shuttle environments ${ }^{3}$ on TPCs treats the bulk energy available for ignition as due to the translational kinetic energy of motion of the striking assembly. The combined mass of the propellant and output assemblies, accelerated at $30 \mathrm{~g}$ through the distance provided when the foam is completely compressed, yields an energy of $0.276 \mathrm{~J}$. Comparison of $0.276 \mathrm{~J}$ value to the BOE $\mathrm{H}_{50}$ test result of $3.6 \mathrm{~J}$ suggests at least an order of magnitude of safety.

A subsequent analysis ${ }^{4}$ was performed that offers a reinterpretation of data by evaluating a critical impact force per unit area sufficient to cause ignition, and comparing it to the force per unit area resulting within the anomalous TPC. The BOE test parameters and results with Hi-Temp are used to determine the critical force per area sufficient to cause ignition. The force is computed as mass $\mathrm{x}$ velocity/change in time, where the change in time is interpreted as an impulse time and arbitrarily chosen to be 0.001 second. The "critical" force is calculated using the mass of the drop weight and velocity at impact. The force per unit area necessary to initiate Hi-Temp is computed using the BOE sample cup cross-sectional area. ${ }^{5}$ The approach is completed by computing the force per area resulting from a worst case impact within an anomalous TPC. This assumes impact by the combined mass of the propellant assembly and output assembly at maximum velocity based on launch acceleration criteria, and over the impact area, now chosen to be the ring-like surface presented by the steel casing of the output assembly. This approach finds there is no margin for safety and that there is a potential for inadvertent initiation of the Hi-Temp by impact. Modern hazard assessment accepts that the concept of an impact force per unit area provides a better correlation to ignition sensitivity for pyrotechnics based on the hot-spot theory of ignition. ${ }^{6}$

\section{Testing Indicated}

The impact force per area analysis is a comparison technique and does not provide a result that can be evaluated against specific impact data. The central assumption regarding the duration of impulse implies that crushing a single grain of Hi-Temp during the hypothesized TPC impact and crushing the $10 \mathrm{mg}$ sample in the BOE tester system sample cup are the same. This is not likely for several reasons involving compaction. Depending on the packing 
fraction, which is not known, the sample will be at least 2 to 3 grains in depth based on density. In a cup, the grains will be compressed on impact with no opportunity to escape. In the case of a grain on the contact area within the TPC, the grain diameter is almost twice the bearing area width. TPC geometry at impact will more likely create a "pinching" effect and with much less confinement than is inherent in standard impact apparatus. The compressing weight or surface will have further to traverse to complete compression in the sample cup as compared with a "pinched" grain. Hence, the duration of the impulse and degree of compression will be different for these two different impact geometries. These observations suggest that better impact information, gained by simulation of the impact geometry, is required before conclusions on Hi-Temp impact sensitivity within a TPC can be drawn.

\section{Approach}

The test approach is to evaluate the worst case impact ignition process within the TPC geometry. Specific assumptions are:

- That a $40 \mathrm{~g}$ launch vibration is possible, and

- Complete foam compression happens, allowing the maximum distance for acceleration,

- $\quad$ The combined weight of the propellant assembly and the output assembly participate in the impact,

- $\quad$ All energy of motion $\left[\mathrm{KE}=0.5 \mathrm{mv}^{2}\right]$ is assumed to be absorbed by the sample,

- Based on the worst case weight and acceleration, the worst case energy is computed to be $0.25 \mathrm{~J}$ (0.184 ft-lbf).

Drop tests will be used to establish the relationship between the worst case impact energy and the energy required to initiate Hi-Temp, and a margin of safety will be established. The test strategy is a modified Bruceton drop test technique applied to single and multigrain scenarios. The modification is to allow the flexibility for experimental exploration as needed because the ultimate behavior of the system is not known.

\section{A. Equipment}

To physically accomplish this, a standard mechanical impact system IMIT (Dynatup ${ }^{\circledR \dagger}$ 8250) is used with the anvil and impactor modified to simulate the "pinch" geometry within the TPC (Fig. 3).
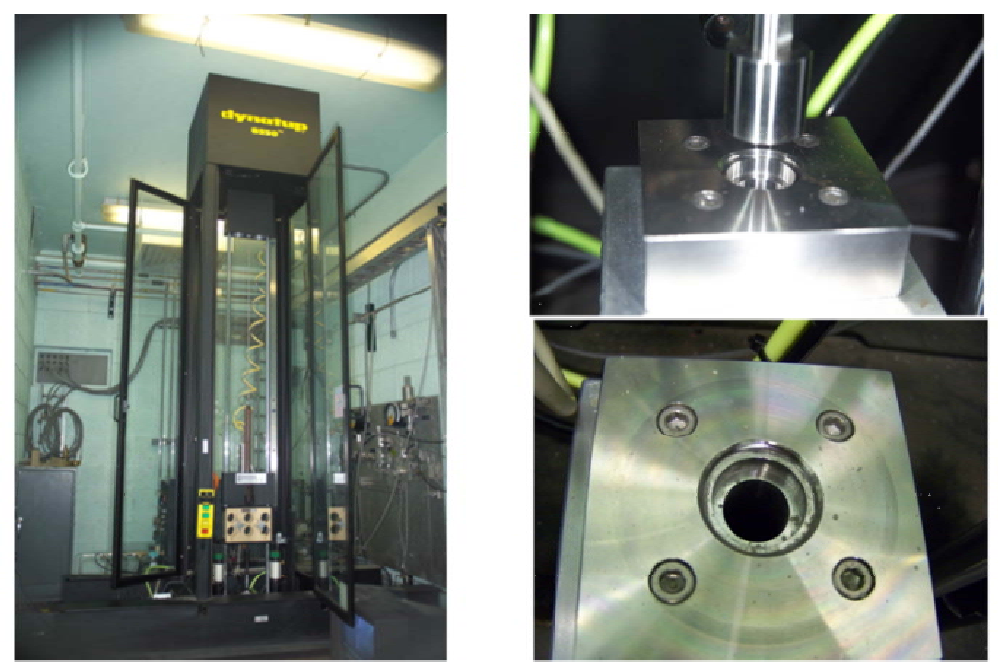

Figure 3. View of mechanical impact system (left), anvil and impactor (upper right) and view into the anvil (lower right)

The impactor is designed to strike grains placed on the "ring" surface inset into the face of the anvil. The diameter of the impactor is identical to the diameter of the output assembly and the width of the "inset" ring matches the surface area available for impact within the TPC. Two different impactors (tups) were used: a "ring tup" and a "flat tup" as depicted by the cross-sectional view in Fig. 4 (not to scale).

\footnotetext{
${ }^{\dagger}$ Dynatup ${ }^{\circledR}$ is a registered trademark of Effects Technology, Inc. Santa Barbara, California.
} 


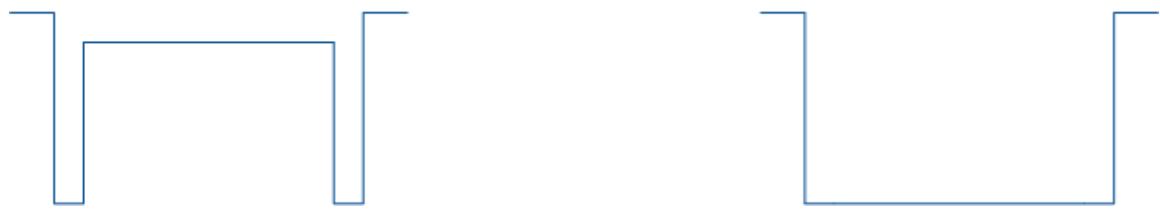

Figure 4. Cross-sectional views of the different impactors used in mechanical impact tests: "ring tup" shown at left and "flat tup" shown at right.

The flat tup was designed to produce the higher energy impacts. The drop height was adjusted to reproduce impact energies of interest.

\section{B. Test Procedure}

The general procedure used for test is described by the following steps:

- $\quad$ Checkout and calibrate IMIT

- $\quad$ Setup IMIT for requested impact energy

- $\quad$ Place propellant grain on impact surface

- $\quad$ Exit test cell, erect barricade

- $\quad$ Start instrumentation, run test

- Inspect for reaction, clean surfaces (as required), remove/dispose of propellant grain/reaction material

- $\quad$ Retest as necessary

\section{Results and Discussion}

Impact tests using single and multiple grains were performed. The general result shown in Table 1 is that no ignitions were observed, even for energies 31 to 132 times in excess of the $0.25 \mathrm{~J}$ (0.184 ft-lbf) computed worst case energy.

Table 1. Impact test results.

\begin{tabular}{ccccccc}
\hline $\begin{array}{c}\text { Test } \\
\text { Series }\end{array}$ & $\begin{array}{c}\text { Number } \\
\text { Of Grains }\end{array}$ & $\begin{array}{c}\text { Average Energy } \\
\text { J (ft-lbf) }\end{array}$ & $\begin{array}{c}\text { Multiplicative } \\
\text { Factor (x 0.25 J) }\end{array}$ & $\begin{array}{c}\text { Number } \\
\text { Of Tests }\end{array}$ & $\begin{array}{c}\text { Tup } \\
\text { Type }\end{array}$ & $\begin{array}{c}\text { Posttest Sample } \\
\text { Description }^{+}\end{array}$ \\
\hline 1 & Single & $7.73(5.7)$ & 30.9 & 11 & Ring & None \\
3 & Single & $19.9(14.7)$ & 79.6 & 11 & Flat & Some Discoloration \\
4 & Single & $24.3(17.91)$ & 97.2 & 10 & Flat & Some Discoloration \\
5 & Multi & $24.6^{*}(18.20)$ & 98.4 & $8^{*}$ & Flat & Almost All Discolored \\
6 & Multi & $25.5(18.83)$ & 102 & 7 & Ring & None \\
2 & Single & $33.1(24.42)$ & 132.4 & 7 & Flat & All Discolored \\
\hline
\end{tabular}

* Two tests performed with a damaged tup that had become sufficiently warped to bind with the anvil ("sideways pinching") and produced a positive result (a report), the only time positive results were obtained. When the tup was re-machined, no further positive results were obtained. Average computed for the 6 successful tests.

$\dagger$ IR spectra taken of the discoloration and compared to IR spectra of untested Hi-Temp were virtually identical indicating that the discoloration was simply the appearance after the grains were mashed.

After impact, the Hi-Temp grains appeared flattened and crushed, and exhibited a non-Newtonian fluid like behavior ('paste' like). IR spectra of pre and posttest examples registered no change and indicated that the change in appearance was not evidence of a minor reaction. 


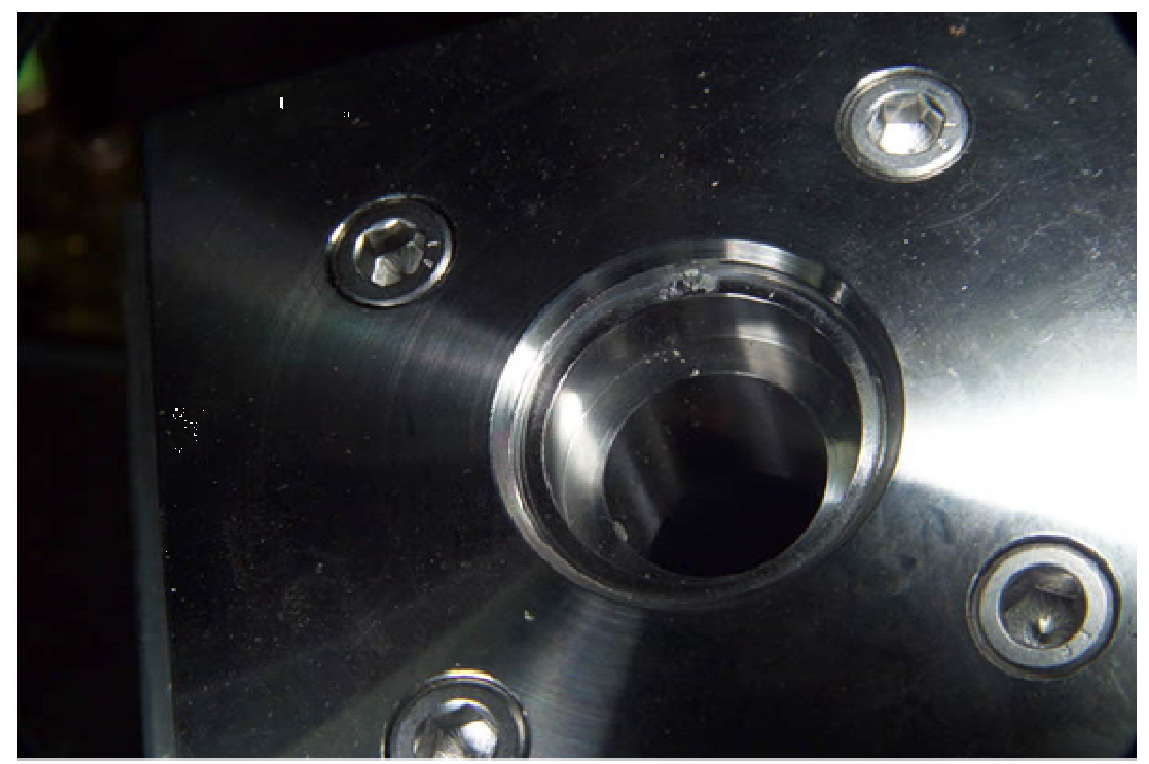

Figure 5. Typical result for a test with a single grain.

Fig. 5 is a posttest photo showing the results of impacting a single grain with an energy 83 times the maximum energy possible within a TPC. The grain appears crushed with a slight discoloration.
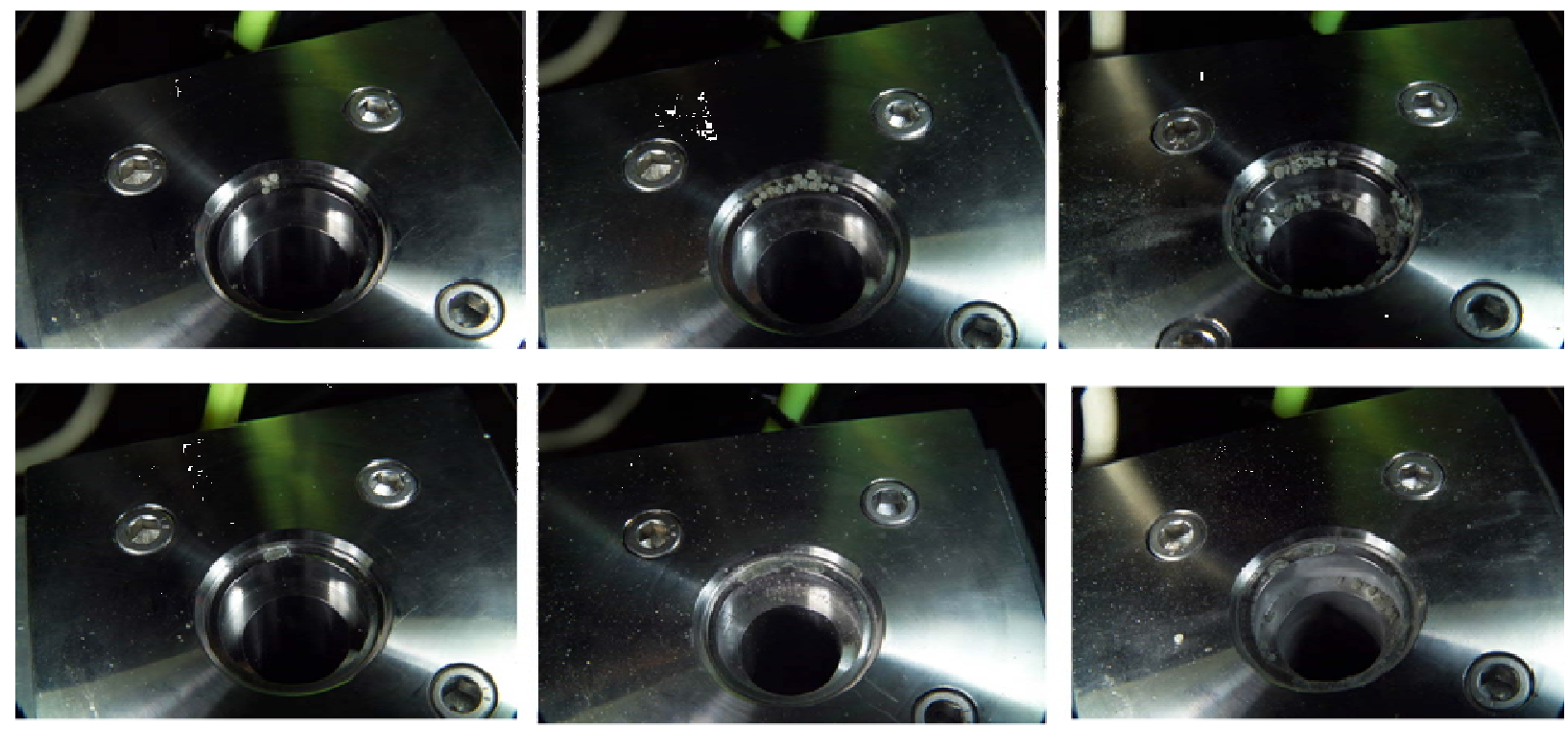

Figure 6. Pre (upper photo) and posttest (lower photo) views for three tests are shown sequentually from left to right.

The photos in Figure 6 again show the discoloration, but no material has been consumed and it is out of view in the "well" of the anvil.

In summary, no reactions occurred under tested impact conditions. The discoloration often noted in tests was not evidence of a reaction as verified by IR spectra taken of pre and posttest samples. Additional factors that may be considered are the effects of friction from the broken epoxy bond and the case walls will slow movement of the assemblies. Different tolerances and presence of unbounded epoxy will prevent the propellant and output assemblies from traveling together. The passages in the system are sufficiently small so that air trapped within gaps will slow movement by creating “damping." The hot-spot ignition mechanism, which is the likely ignition mechanism thought to act in this scenario, is not well supported due to the small number of grains in contact, and also by the geometrical fact that the width of impacting surfaces is less than a nominal grain diameter and hence cannot crush an entire grain. The Hi-Temp in grain form impacted in TPC geometry showed stability in both single and multiple grain tests 
for impacts far in excess, approximately 83 to 136 times, of levels that hypothetically could form within a TPC under launch vibration conditions.

\section{Conclusion}

Based on these results, the probability of impact-induced ignition is extremely small. The point of ignition for the TPC geometry was not established, providing a basis for an assertion that the safety factor is greater than several orders of magnitude even if conditions within the TPC achieve the computed theoretical $0.25 \mathrm{~J}$ (0.184 ft-lbf) maximum impact energy arising from a $40 \mathrm{~g}$ launch environment, which is by itself a very unlikely occurrence. In conclusion, the worst case scenario is unrealistic and mechanical impact will not cause ignition.

\section{Acknowledgments}

The authors would like to thank Stephen McDougle, Ralph Lucero, Chris Keddy, Chris Wojtko, and Tommy Yoder of the WSTF Laboratories Department; and Mike Mannon and Richard Madrid of the WSTF Materials 800 Area Test Facility.

\section{References}

${ }^{1}$ Piatkowksi, E., Hazard Testing of HI-TEMP Smokeless Powder Sample (ESD Sensitivity, BOE Impact Sensitivity, and BAM Friction Sensitivity Tests). UTEC Corporation, R\&D Laboratory, Laboratory Report No. 09011, Riverton, Kansas 66770, January 30, 2009.

${ }^{2}$ Gibbs, T. R., and Popolato, A., LASL Explosive Property Data. University of California Press, Berkeley, 1980.

${ }^{3}$ McDonald, S., Potential Inadvertent Initiation of the Thruster Pressure Cartridge due to Impact Sensitivity of Loose HiTemp Propellant, UPCO, February 3, 2009.

${ }^{4}$ Gunderson, K., Approach to Calculating the Inherent Risk of HI-TEMP Premature Initiation due to Internal Impact, NASA Marshall Space Flight Center, Huntsville, Alabama. February 9, 2009.

${ }^{5}$ MIL-STD-1751A, Method 1011, Safety and Performance Tests for the Qualification of Explosives, December 2001.

${ }^{6}$ Field, J. E., Hot Spot Ignition Mechanisms for Explosives. American Chemical Society 1992, v. 25, pp. 489-496. 


\section{Problem Description}

- Launch vehicles use pyrotechnic thruster pressure cartridges (TPCs) for aeroshell separation

- Units undergoing acceptance testing had nominal performance, but NDE indicated that:

- Internal assemblies moved during shock testing (failed epoxy bond)

- Grains of second stage pyrotechnic, Hi-Temp ${ }^{\mathrm{TM}}$, were loose

- Mission timeline precluded fabrication of a new lot

- Concerns raised - Could launch

Photo of TPC Unit
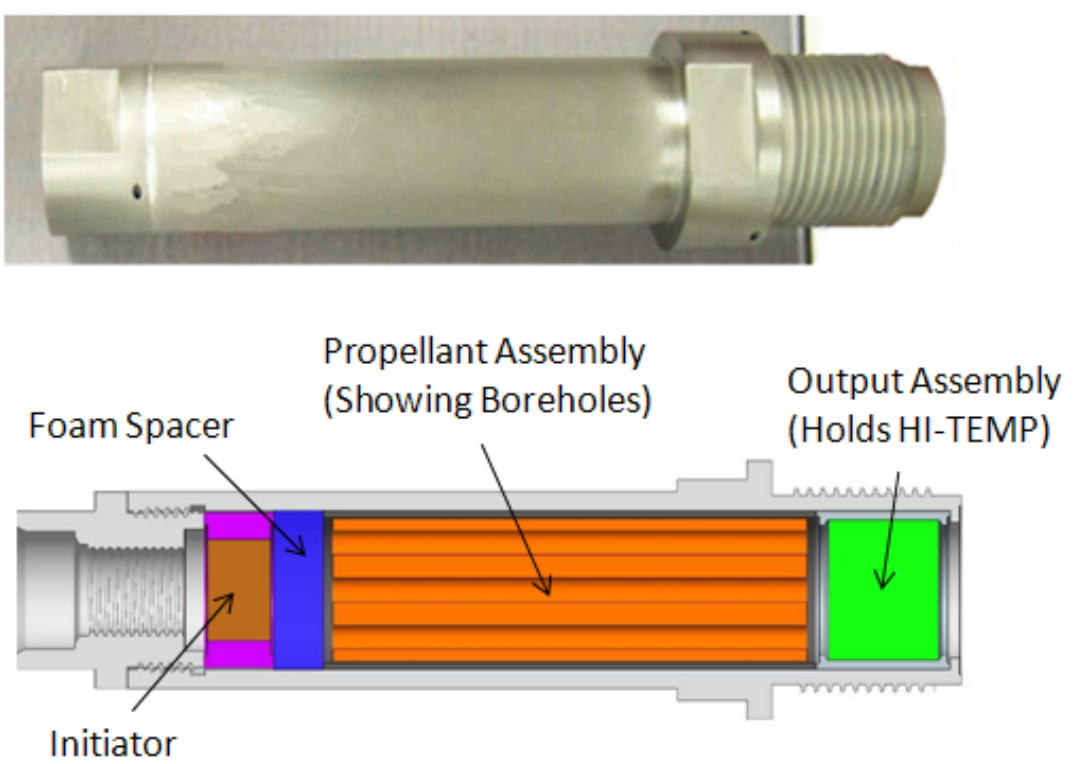

Diagram of TPC Internal Components vibration induce sufficient motion in loose internal subcomponents to cause premature ignition of Hi-Temp by mechanical impact?
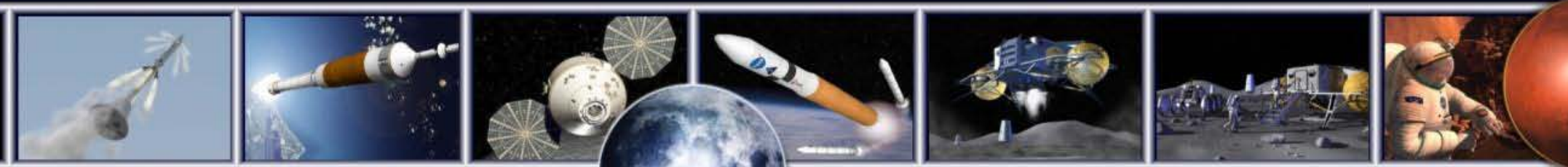


\section{Mechanical Impact Analysis}

- Existing data and concerns

- Critical drop energy for Hi-Temp (BOE test): $3.6 \mathrm{~J}$

- Applies a $3.63 \mathrm{~kg}$ weight to a $10 \mathrm{mg}$ sample in a .2-in. diameter cup. Drop height is varied over 1-in. intervals to determine $\mathrm{H}_{50}$ (ignition $50 \%$ of the time)

- KE analysis for worst case acceleration and mass within TPC: $0.276 \mathrm{~J}$

- This is a critical "bulk" energy: suggests a $>10$ safety factor

- Hot spot initiation theory considers the impact force per unit area

- Force is concentrated between the rim area of the output assembly and the case lip

- "Critical" force is obtained from BOE data as velocity over $\Delta \mathrm{t}$ where $\Delta \mathrm{t}$ is assumed to be $1 \mathrm{~ms}$

- Mass of output and propellant assemblies act together under 3 sigma acceleration (40 g)

- Impact force based on KE of motion is computed using the $1 \mathrm{~ms}$ assumption

- The comparison shows equivalent psi values implying no safety factor
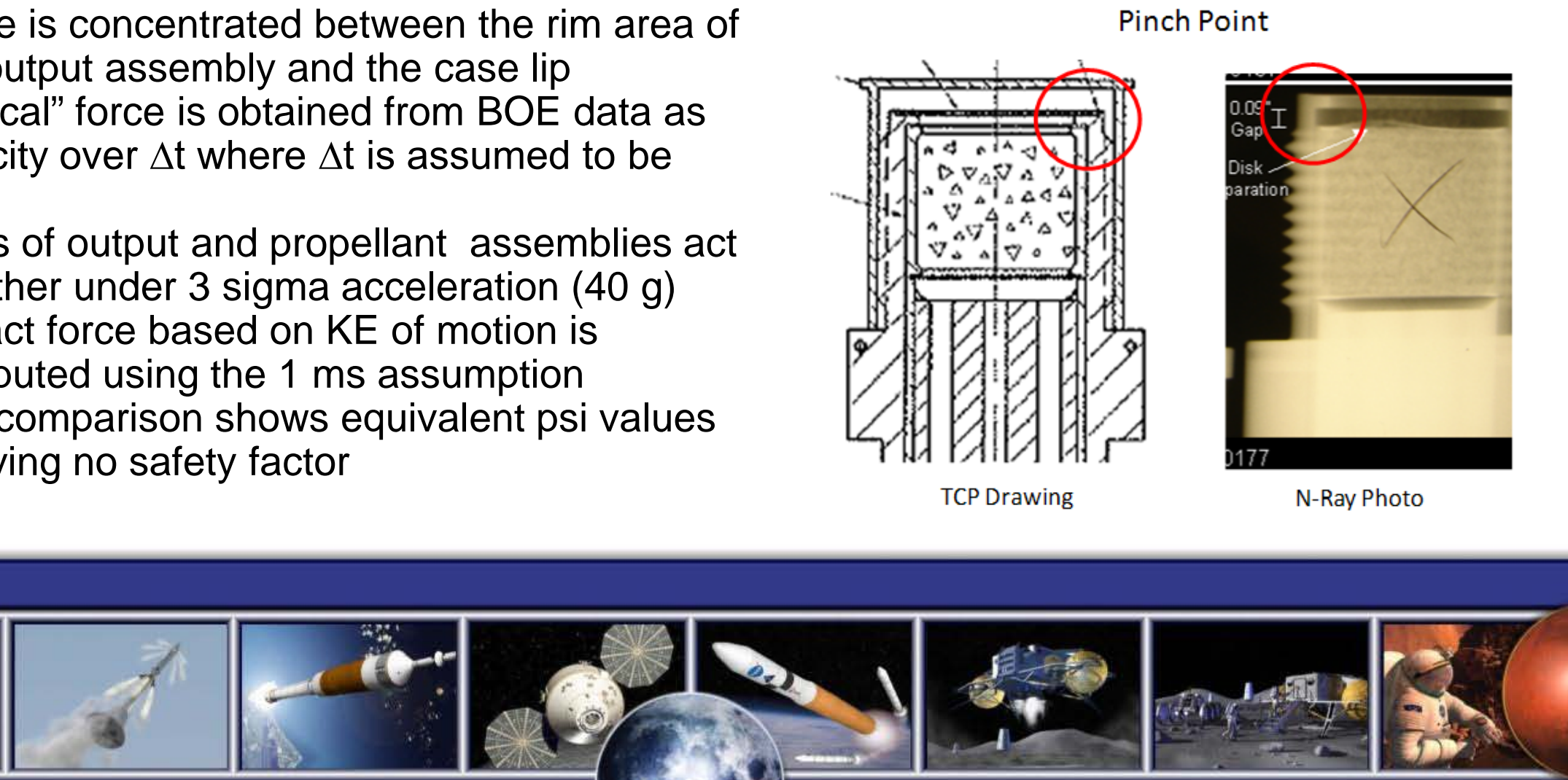


\section{New Testing Indicated}

- The impact force per area analysis does not provide a result that can be compared to test data

- Assumptions made in the analysis imply:

- Time duration to crush a single grain is the same as the duration to crush $10 \mathrm{mg}$ in a sample cup

- Impact and confinement within the BOE sample cup is equivalent to that of TPC geometry

- TPC impact area width is half that of grain diameter: incomplete confinement ... more of a "pinch" effect

- Impact testing with an impactor-anvil to duplicate the TPC impact geometry is indicated

- Evaluate impact energy using a modified Bruceton test method and compare to a worst case (max mass @ $40 \mathrm{~g}$ ) impact (0.25 J)
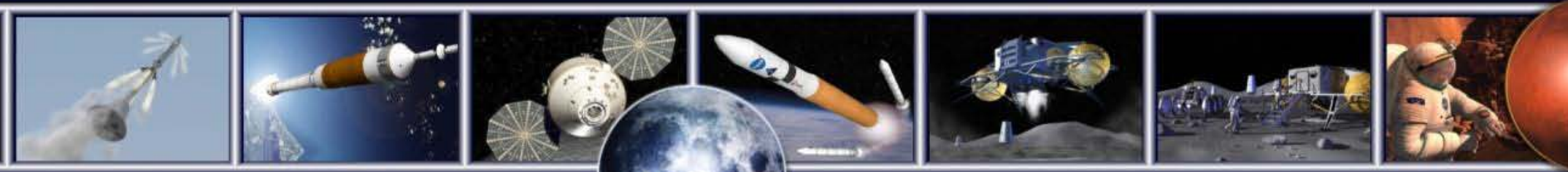


\section{Tester, Impactor, and Anvil}
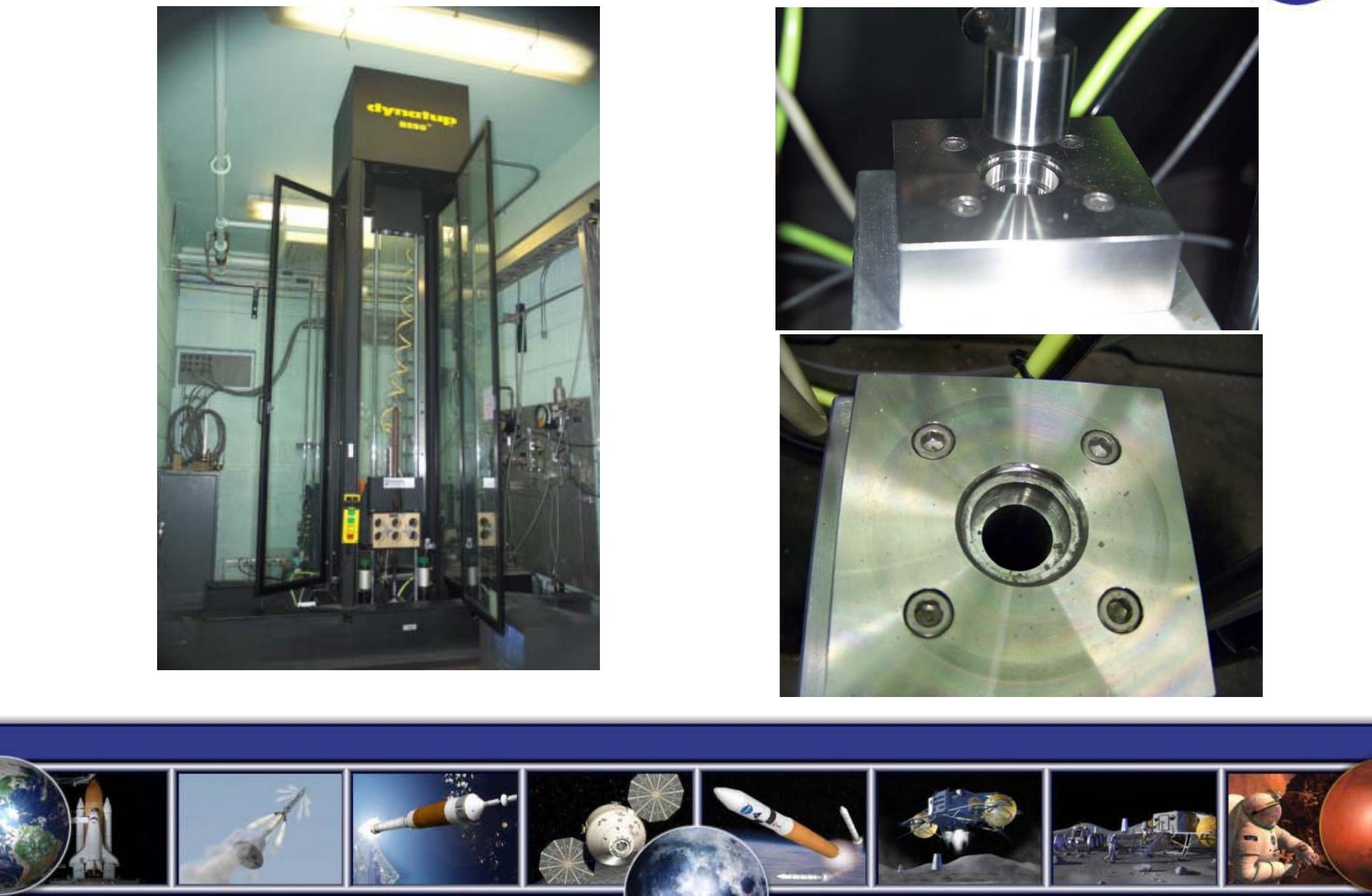


\section{Impact Test Results}

- Modified Bruceton drop test technique applied to single and multigrain scenarios

- No discernible change in appearance at $7.73 \mathrm{~J}(5.7 \mathrm{ft}-\mathrm{lbf}) 31 \mathrm{x}$ the maximum computed impact level $(0.25 \mathrm{~J})$

- Some minor change in color at very high levels, $25-33.9 \mathrm{~J}(18.1-25 \mathrm{ft}-\mathrm{lbf})$

- Post-impact analysis of Hi-Temp grains

- Typical result: Material was flattenedlcrushed, no reaction, some non-Newtonian fluid-like behavior ('paste' like)

- IR spectra measurements of virgin and impacted material taken to look for chemical change at 11 and $13.3 \mathrm{~J}$ (15 and $18 \mathrm{ft}-\mathrm{lbf}$ levels) or 83 to 100 times maximum computed level

- No significant change in spectra noted

- Hi-Temp in grain form impacted in TPC geometry showed stability in both single and multiple grain scenarios far in excess, $>83$ to 136 times, of the impact levels that could form in a launch scenario
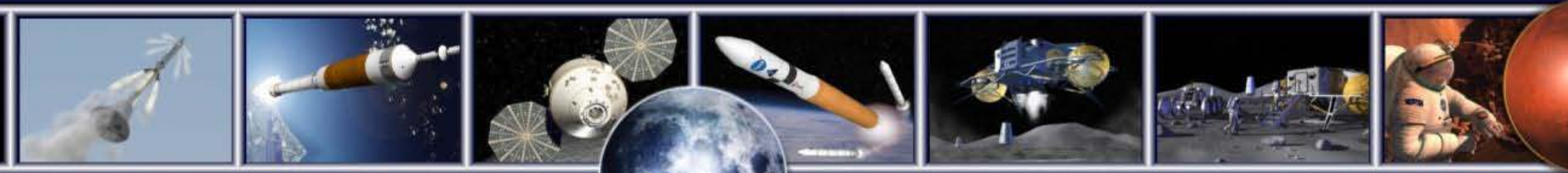
- 3 sigma acceleration ( $30 \mathrm{~g}$ to $40 \mathrm{~g}$ ) is statistically unlikely and is unlikely to act through the entire path until impact

- Friction from epoxy and the TPC case walls will slow assemblies

- Different tolerances and presence of unbonded epoxy will prevent the propellant and output assemblies from traveling together

- Compression of air within the gap will slow velocity of assemblies

- Hot-spot ignition mechanism unlikely due to small number of grains in contact and impact that cannot crush an entire grain (width of impacting surfaces < grain diameter)

\section{Conclusion: Worst case is unrealistic Mechanical impact will not cause ignition}
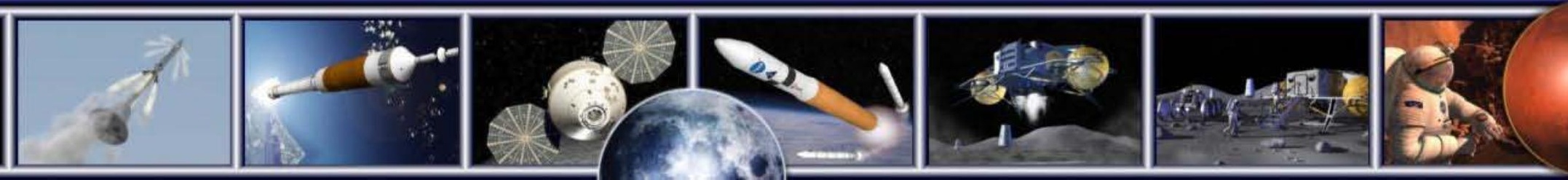


\section{Conclusions}

- Based on theoretical and test considerations, mechanical movement of inner TPC components due to failed epoxy bonds does not appear capable of sufficient mechanical impact energy to cause uncommanded ignition of Hi-Temp during flight

- The analysis supports a risk-informed assessment process

- Quantitative risk assessment or determination of a data-based safety factor would still require additional information

- Fabrication of TPCs that prevent movement of the propellant assembly or release of Hi-Temp would preclude the need for mechanical impact analysis
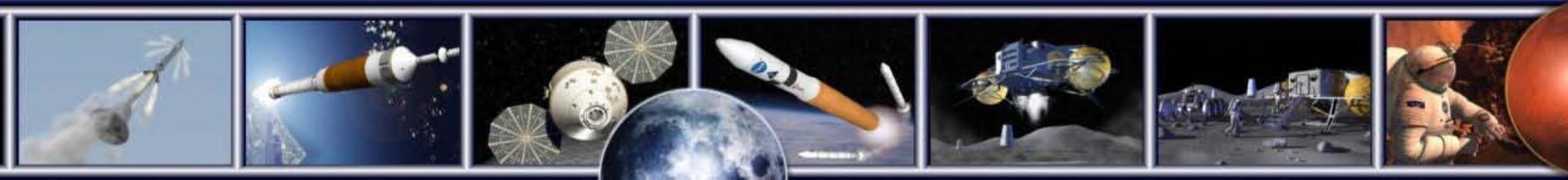


\section{For More Information}

- For more information, please contact:

- Regor Saulsberry (575) 524-5518 or email: regor.l.saulsberry@nasa.gov

- Stephen Woods (575) 524-5607 or email: stephen.s.woods@nasa.gov

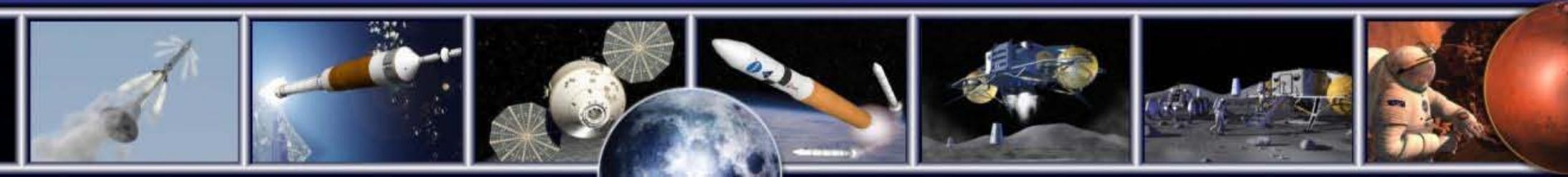

